\title{
Thermo-electrical stability in an electrode
}

\author{
A. $\operatorname{LACEY}^{1} \dagger$ \\ ${ }^{1}$ Heriot-Watt University \\ (Communicated to MIIR on 23 June 2021)
}

Study Group: ESGI 31. Southampton 22-27 March 1998

Communicated by: David Allwright

Industrial Partner: Elkem

Team Members: Harold Hanche-Olsen, Norwegian University of science and Technology; Peter Howell, University of Oxford; Andrew Lacey, Heriot-Watt University; Mark Peletier, Eindhoven University of Technology; Donald Schwendeman, Rensselaer Polytechnic Institute; Warren Smith, University of Nottingham; Richard Tew, University of Nottingham; Jonathan Wattis, University of Nottingham.

Industrial Sector: Materials processing

Tools: Partial differential equations, Stability analysis, Heat equation, Dynamics

Key Words: Heat flow, Thermo-electrical instability, Conductivity

MSC2020 Codes: 78, 80

\section{Summary}

The operation of an electrode under transient conditions, in particular the possibility for instabilities, is considered. Various simplified models are analysed to determine whether or not instabilities might occur, and if so, over what parameter range.

$\dagger$ Address for Correspondence: A.A.Lacey@hw.ac.uk 


\title{
Thermo-Electrical Stability in
}

\author{
an Electrode - Elkem
}

\section{Introduction}

The problem considered was the operation of an electrode, such as a Søderberg or Persson electrode, under transient conditions. In particular, the possibility of potential instabilities in the steady-states was discussed. It is important to know whether or not, after a change from normal operating conditions, a furnace and its electrode will return to their earlier equilibrium-if they do not then there is a possibility of a dangerous situation arising.

To avoid extensive computations associated with the full thermo-electrical problem, attention was focused on a single electrode with a specified electric current flowing through it. This lead to a simpler coupled elliptic-parabolic problem, for the electric potential and temperature. The geometry and boundary conditions for this simpler model, together with current flow and cooling conditions which varied according to position on the electrode surface (e.g., above the clamp, at the clamp, or below the clamp), meant that only a range of even simpler model problems were considered. These mathematical models were analysed to determine whether or not instabilities in their steady-states might occur and, if so, over what sort of parameter range (corresponding to possible novel furnace designs or operations) they might occur, and lead to potentially dangerous situations in practice.

Although the current in a real elctrode is alternating, for simplicity only models for direct-current flow were considered in detail. Some comments regarding the $\mathrm{AC}$ situation, however, are made at the end of this report.

\section{Basic Model}

The heat and electric-current flow through only a single medium, the carbon paste constituting the bulk of the electrode, were considered. The steel in a Søderberg electrode and the graphite in a Persson electrode were ignored. 
The appropriate heat equation is then

$$
\rho C\left(\frac{\partial T}{\partial T}+U \frac{\partial T}{\partial z}\right)=\kappa \nabla^{2} T+|\boldsymbol{j}|^{2} / \sigma
$$

where $\rho, C, \kappa$ are the density, specific heat, and thermal conductivity of the carbon paste, all regarded as constants (it was assumed that they do not vary much compared to the the electrical conductivity), $z$ is the distance along the electrode measured vertically downwards, $U$ is the velocity of the electrode in the $z$ direction, $j$ is the current density and $\sigma$ is the electrical conductivity. The electrical conductivity was assumed to be a function of temperature, $\sigma=\sigma(T)$, although, in fact, it might also depend upon baking time. The electrical conductivity of the carbon paste, as it is baked, seems to be fairly well modelled by

$$
\begin{gathered}
\sigma=\sigma_{\infty}\left(1+\exp \left(\frac{T_{c}-T}{T_{d}}\right)\right)^{-1} \\
T_{d}=65^{\circ} \mathrm{C}, \quad T_{c}=775^{\circ} \mathrm{C}, \quad \sigma_{\infty}=32000 \Omega^{-1} \mathrm{~m}^{-1} .
\end{gathered}
$$

Note that this relationship holds only if the temperature of the carbon paste is rising and if it should ever drop, the conductivity would remain approximately constant until the temperature is raised above the previous maximum.

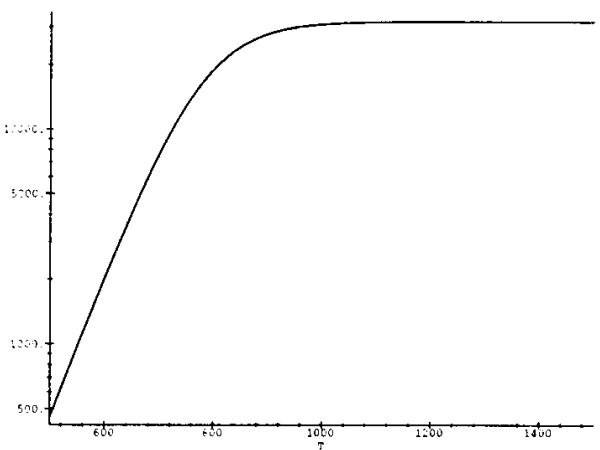

(a)

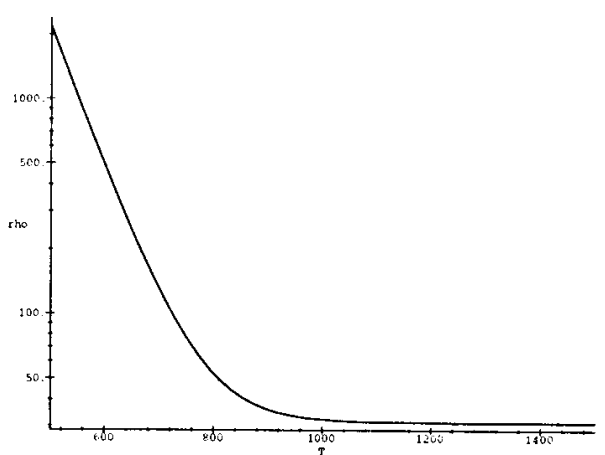

(b)

Figure 1: (a) Conductivity, $\left(\Omega^{-1} \mathrm{~m}^{-1}\right)$ and (b) resistivity $(\mu \Omega \cdot \mathrm{m})$ as functions of temperature. Compared to measured data, the bend in the curve (a) is a bit too sharp and the level part somewhat too flat.

Calculations done during the 1997 ESGI suggest that the Péclet number for this problem is $O(1)$, so convection and conduction are equally important. In much of what follows, however, convection is ignored as it does not appear to greatly affect the qualitative behaviour of the solutions of this problem. 
The electric current is given by Ohm's law (neglecting the interaction of the magnetic field with the small velocity):

$$
j=\sigma E
$$

and, for direct current, an electric potential $\varphi$ exists so that

$$
\boldsymbol{E}=-\nabla \varphi
$$

so that

$$
j=-\sigma \nabla \varphi
$$

For (approximate) charge neutrality,

$$
\nabla \cdot j=0
$$

and

$$
\nabla \cdot(\sigma \nabla \varphi)=0
$$

It is sometimes more convenient to write the heat equation as

$$
\rho C\left(\frac{\partial T}{\partial t}+U \frac{\partial T}{\partial z}\right)=\kappa \nabla^{2} T+\sigma|\nabla \varphi|^{2} .
$$

\section{Rapidly-Varying Conductivity Model}

It was noted that under normal present-day operation, most of the current would be flowing through a region where the temperature was high enough for $\sigma(T)$ not to be varying significantly, while $\sigma(T)$ does grow something like exponentially fast for lower temperatures. Sketches of conductivity and resistivity (to the paste) against temperature are shown in Figure 1.

Indeed, where $\sigma$ grows, it can have quite sensitive dependence upon temperature (with $T$ varying over $O\left(10^{3}{ }^{\circ} \mathrm{C}\right.$ ), see (2)). Since, having $\sigma$ nearly constant would appear unlikely to lead to instability, which was what was being sought, it was supposed that there might be a significant region of the electrode where $T$ was such that $\sigma(T)$ was rapidly growing, say something like $\sigma=e^{\lambda T}$ with $\lambda$ "large".

To satisfy (4), it is natural to try a WKB expansion for $\varphi$, so $\varphi \sim$ $\Phi(\mathbf{x}, t) e^{\lambda \Phi(x)}$, and it transpires that

$$
\varphi \sim \Phi(\mathbf{x}, t) e^{-\lambda T}
$$

for some function $\Phi$. Substitution into (5) gives

$$
e C \frac{\partial T}{\partial t}+U \frac{\partial T}{\partial z} \sim \kappa \nabla^{2} T
$$


to leading order $T$ satisfies a linear heat equation.

Although these approximations are likely to fail near the surface of the electrode, especially in the vicinity of the cooled copper clamps which supply the current, this approach does not appear to indicate a mechanism for instability.

\section{The Lumped-Resistor Model}

We now consider a very simple representation of the electrode as a single resistor with uniform temperature $T(t)$. The resistance depends upon temperature, $R=R(T)$, and, for a specified voltage $V, T$ satisfies an ODE of the form

$$
\frac{d T}{d t}=\frac{A V^{2}}{R}-B\left(T-T_{a}\right)
$$

where the cooling term $B\left(T-T_{a}\right)$ represents heat loss by convection and radiation. Likewise, for fixed current $I$,

$$
\frac{d T}{d t}=A I^{2} R-B\left(T-T_{a}\right)
$$

Taking $R$ to have the form indicated by Figure 1 (b), e.g.,

$$
R(T)=R_{\infty}\left(1+\exp \left(\frac{T_{c}-T}{T_{d}}\right)\right)
$$

it is clear that (7) has a unique steady state, and this is globally asymptotically stable, whereas (6) has, if $T_{d}<T_{c} / 2$, a range of $V$ for which there are three steady states; for smaller or larger $V$ the equilibria are again unique and globally asymptotically stable. Should $V$ be such that (6) has multiple steady solutions, it is clear that the largest and smallest are stable but the middle one is unstable, see Figure 2.

Introducing dimensionless variables $T^{*}, I^{*}, V^{*}$ and $t^{*}$ by

$$
T=T_{a}+T_{d} T^{*}, I=\sqrt{ }\left(B T_{d} / R_{\infty}\right) I^{*}, V=\sqrt{ }\left(B T_{d} R_{\infty}\right) V^{*}, t=t^{*} / B
$$

inserting these into the above model and dropping the stars we get

$$
\frac{d T}{d t}=V I-T, \quad V=\left(1+e^{\tilde{T}-T}\right) I
$$

where

$$
\tilde{T}=\frac{T_{c}-T_{a}}{T_{d}}
$$



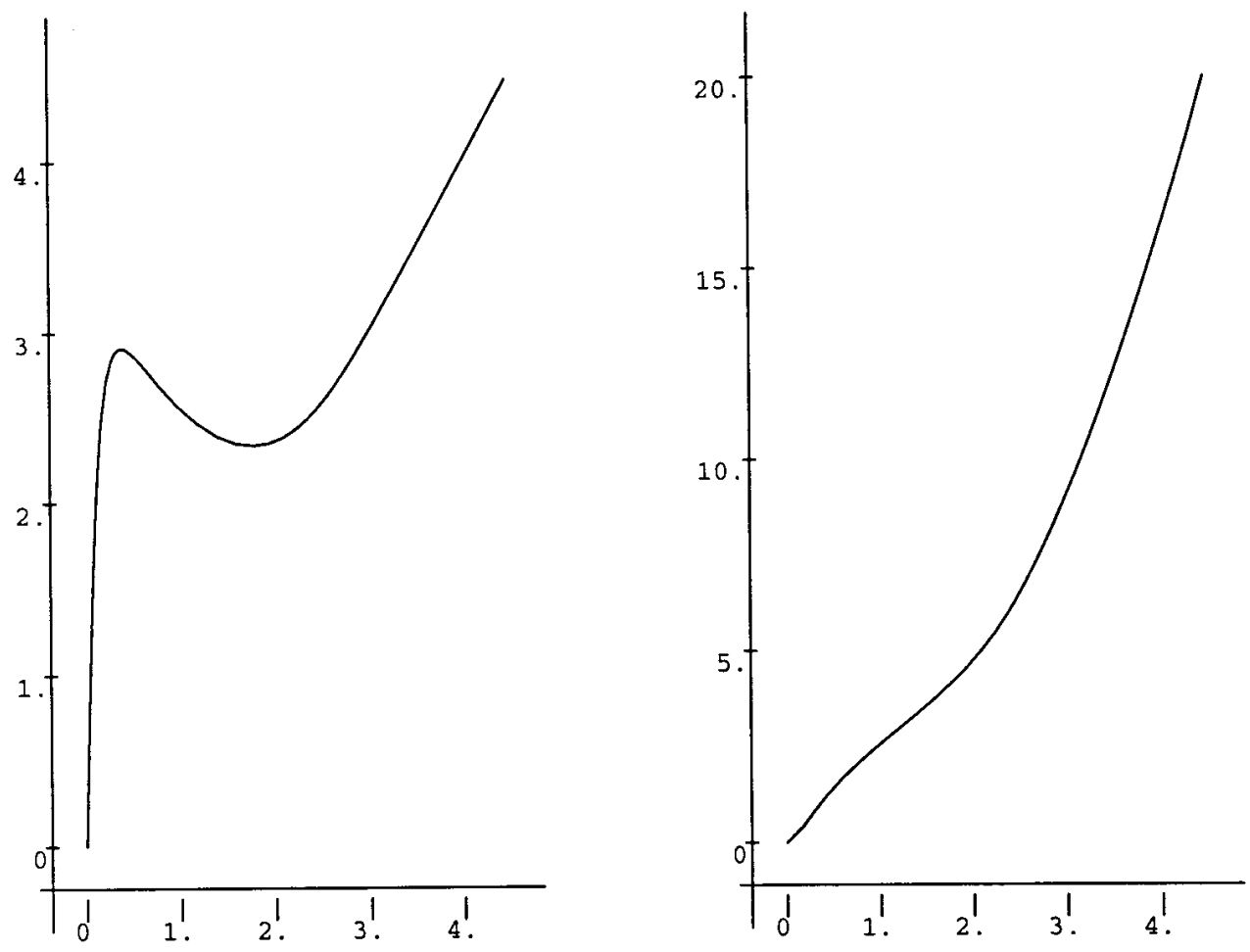

Figure 2: (Left) voltage versus current and (right) temperature versus current at thermal equilibrium $(d T / d t=0)$ for the single-resistor model with $\tilde{T}=3$.

Figure 2 shows that, if the equilibrium states of the single resistor are plotted, voltage can be a non-monotone function of current.

A refinement of this model is essentially to split the electrode into two parallel resistors:

$$
\frac{d T_{1}}{d t}=A I_{1}^{2} R\left(T_{1}\right)-B\left(T_{1}-T_{a}\right), \quad \frac{d T_{2}}{d t}=A I_{2}^{2} R\left(T_{2}\right)-B\left(T_{2}-T_{a}\right),
$$

ignoring thermal linkage between the two parts, or

$$
\begin{aligned}
& \frac{d T_{1}}{d t}=A I_{1}^{2} R\left(T_{1}\right)-B\left(T_{1}-T_{a}\right)-C\left(T_{1}-T_{2}\right), \\
& \frac{d T_{2}}{d t}=A I_{2}^{2} R\left(T_{2}\right)-B\left(T_{2}-T_{a}\right)+C\left(T_{1}-T_{2}\right),
\end{aligned}
$$

including such effects, with

$$
I_{1}+I_{2}=J=\text { specified current, }
$$

and $I_{1} R\left(T_{1}\right)=I_{2} R\left(T_{2}\right)=$ voltage drop down the electrode. The sizes of $A, B, C$ are indicated by the properties of the paste, dimensions of the electrode and operating conditions. 
From Figure 2 it is clear that an asymmetric equilibrium can be found; different currents pass through the two resistors although they experience identical potential drops. Figure 3 shows the currents $\left(I_{1}, I_{2}\right)$ corresponding to stable states in this model. For a given total current $I$, the line $I_{1}+I_{2}=I$ may contain two equilibria in addition to the one on the diagonal. In those cases, the equilibrium on the diagonal is unstable, while the two off-diagonal ones are stable. On the other hand, when the only equilbrium is on the diagonal, it is stable.

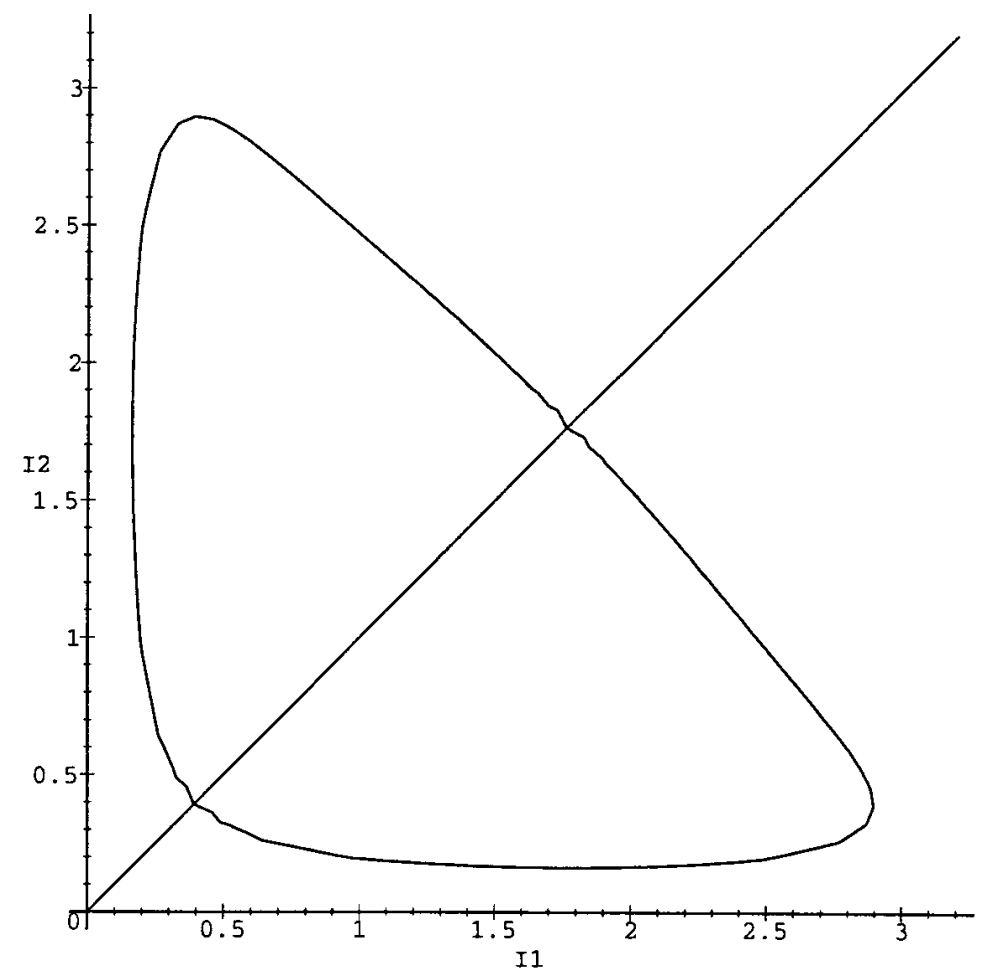

Figure 3: Locus of stability for the two-resistor model with no heat transfer between resistors $(\beta=0)$.

Using the same scaling as for the single-resistor model, and after dropping the stars, the two-resistor model with internal heat transfer is

$$
\begin{aligned}
\frac{d T_{i}}{d t} & =R\left(T_{i}\right) I_{i}^{2}-T_{i}-\beta\left(T_{i}-T_{3-i}\right) \quad(i=1,2), \\
I_{i} & =\frac{R\left(T_{i}\right) I}{R\left(T_{1}\right)+R\left(T_{2}\right)} \\
R(T) & =\left(1+e^{\tilde{T}-T}\right) .
\end{aligned}
$$


We can investigate the stability of a symmetric equilibrium of this model by linearising the system in the neighbourhood of the equilibrium point. The derivative of the right-hand side of our system with respect to $\left(T_{1}, T_{2}\right)$ is the matrix

$$
\left(\begin{array}{cc}
\frac{R_{2}^{2}\left(R_{2}-R_{1}\right)}{\left(R_{1}+R_{2}\right)^{3}} R_{1}^{\prime}-(1+\beta) & \frac{2 R_{1}^{2} R_{2}}{\left(R_{1}+R_{2}\right)^{3} R_{2}^{\prime}}+\beta \\
\frac{2 R_{2}^{2} R_{1}}{\left(R_{1}+R_{2}\right)^{3} R_{1}^{\prime}}+\beta & \frac{R_{1}^{2}\left(R_{1}-R_{2}\right)}{\left(R_{1}+R_{2}\right)^{3}} R_{2}^{\prime}-(1+\beta)
\end{array}\right)
$$

where $R_{i}=R\left(T_{i}\right)$ and $R_{i}^{\prime}=R^{\prime}\left(T_{i}\right)$.

In particular, the linearisation at a symmetric equilibrium point $\left(T_{1}=\right.$ $\left.T_{2}=T, R_{1}=R_{2}=R(T), I_{1}=I_{2}=I / 2, V=R(T) I / 2\right)$ is given by the matrix

$$
\left(\begin{array}{cc}
-1-\beta & \frac{R^{\prime}}{4}+\beta \\
\frac{R^{\prime}}{4}+\beta & -1-\beta
\end{array}\right)
$$

whose eigenvalues are $-1+R^{\prime} / 4$ and $-1-2 \beta-R^{\prime} / 4$. Bearing in mind that $R^{\prime}<0$, we conclude that this equilibrium is stable if $R^{\prime}>-4(1+2 \beta)$ and unstable if $R^{\prime}<-4(1+2 \beta)$.

As might be expected, a larger value of $\beta$ tends to stabilise the model. This can also be seen from the phase diagrams in Figure 4, which show the equilibrium undergoing a pitchfork bifurcation as $\beta$ varies.

\section{The Ring Model}

We now think of the electrode as a thin cylinder, perhaps with an insulating middle, through which the current passes in the axial direction. (This model might be justified if a conducting zone near the bottom of the clamps lies outside and below paste which is effectively insulating and above paste which is so well baked it can be thought of as a perfect electrical conductor.) If we keep the total current fixed at a value $I$ and the temperature is assumed to vary only in the azimuthal $(\theta)$ direction, we obtain a model of the form

$$
\frac{\partial T}{\partial t}=D \frac{\partial^{2} T}{\partial \theta^{2}}+\frac{I^{2} K(T)}{\left(\int_{0}^{2 \pi} K(T) d \theta\right)^{2}}-\alpha T
$$

Here $K(T)=1 / R(T)$ and $\int_{0}^{2 \pi} K(T) d \theta$ represents the total conductivity of the electrode. Now we linearise this around an equilibrium at a constant 

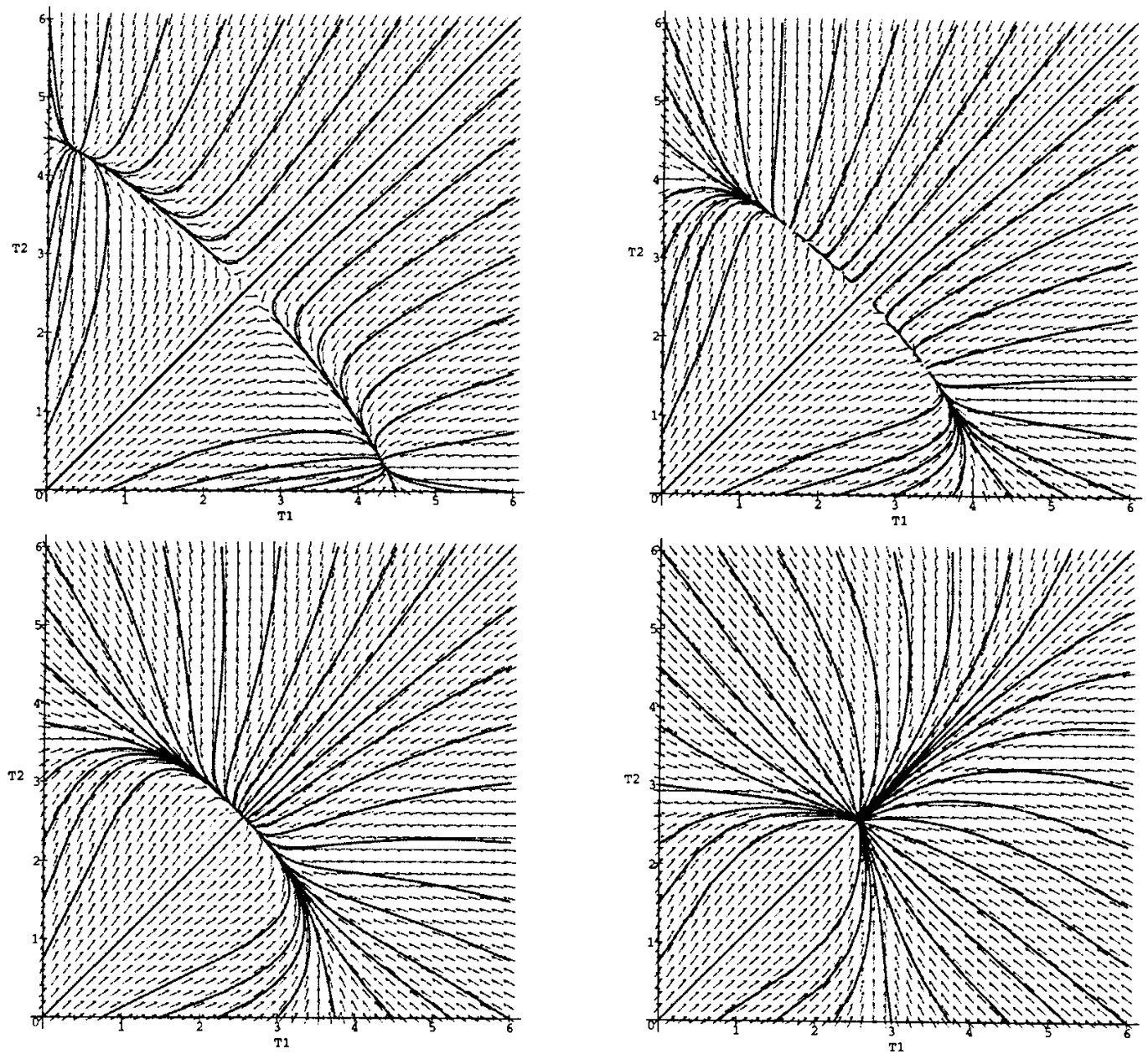

Figure 4: Phase diagram for the two-resistor model. The two axes are $T_{1}$ and $T_{2}$; the total current is $I=2$, and $\tilde{T}=3 . \beta$ varies between the figures (in reading order) as follows: $\beta=0, \beta=0.2, \beta=0.5, \beta=1.5$.

temperature $T_{0}$. That is, we put $T=T_{0}+S$ and work to the lowest order in $S$ :

$$
\frac{\partial S}{\partial t}=D \frac{\partial^{2} S}{\partial \theta^{2}}+\frac{I^{2} K^{\prime}\left(T_{0}\right) S}{\left(2 \pi K\left(T_{0}\right)\right)^{2}}-2 \frac{I^{2} K\left(T_{0}\right) \int_{0}^{2 \pi} K^{\prime}\left(T_{0}\right) S d \theta}{\left(2 \pi K\left(T_{0}\right)\right)^{3}}-\alpha S .
$$

We can solve this by putting $S=e^{\gamma t} \sin \theta$ where

$$
\gamma=-D+\frac{I^{2} K^{\prime}\left(T_{0}\right)}{\left(2 \pi K\left(T_{0}\right)\right)^{2}}-\alpha=-D-\alpha-\frac{R^{\prime}\left(T_{0}\right) I^{2}}{4 \pi^{2}}
$$

and so the equilibrium is unstable if

$$
R^{\prime}\left(T_{0}\right)<-4 \pi^{2} \frac{D+\alpha}{I^{2}}
$$




\section{A One-Dimensional Model}

The first representation considered in this section is related to those of the previous two sections. Imagine that the electric current flows from one end of the electrode to the other and that heat flow in the horizontal direction is insignificant. Then, in the absence of convection (ignoring the electrode motion),

$$
\frac{\partial T}{\partial t}=D \frac{\partial^{2} T}{\partial z^{2}}+\frac{V^{2} R(T)}{\mathcal{A} \rho C\left(\int_{o}^{L} R(T) d z\right)^{2}}
$$

that is,

$$
\frac{\partial T}{\partial t}=D \frac{d^{2} T}{d z^{2}}+\frac{V^{2} / \sigma(T)}{\rho C\left(\int_{0}^{L} d z / \sigma(T)\right)^{2}},
$$

if voltage $V$ is specified, and

$$
\frac{\partial T}{\partial t}=D \frac{\partial^{2} T}{\partial z^{2}}+\frac{I^{2} R(T)}{\mathcal{A} \rho C}
$$

if current $I$ is fixed. In these equations

$$
D=\text { thermal diffusivity }=\kappa / \rho C
$$

$L=$ length of the electrode (distance between clamp and base),

$$
R=\text { resistance per unit length }=1 / \mathcal{A} \sigma(T)
$$

and

$$
\mathcal{A}=\text { cross-sectional area. }
$$

We suppose that thermal boundary conditions of the form

$$
\frac{\partial T}{\partial z}=0 \text { at } z=L \text { (base of electrode) }
$$

and

$$
\alpha \frac{\partial T}{\partial z}=T \text { at } z=0 \text { (clamp) }
$$

apply.

The co-efficient $\alpha$ might be taken to be of the size of the clamp or the radius of the electrode. (The zero temperature has been taken as clamp or initial electrode temperature, or ambient temperature.)

The standard equation (11), with $R$ a positive but decreasing function of $T$, has a unique stationary solution, and this is globally asymptotically stable. The equation (10), (derived from (11) on using $V=I \int_{0}^{L} R d z$, see 
[1] and [3]) has steady solutions given in terms of the two-point boundary problem

$$
\frac{d^{2} T}{d z^{2}}+\lambda R(T)=0,0<z<L, \quad \alpha \frac{d T}{d z}=T(0), \quad g \frac{d T}{d z}(L)=0
$$

with

$$
V^{2}=\rho C \lambda D\left(\int_{0}^{L} R(T) d z\right)^{2}
$$

From (12), $\int_{0}^{L} R d z=\frac{1}{\lambda} \frac{d T}{d z}(0)$ and $\left(\frac{d T}{d z}(0)\right)^{2}=2 \lambda \int_{T(0)}^{T(L)} R(T) d T$, so

$$
V^{2}=4 \kappa \int_{T(0)}^{T(L)} R(T) d T
$$

The problem (12) (which is the steady version of (11)) has a unique solution for all $\lambda>0$ and $T(0)+L \frac{d T}{d z}(0)=(1+L / \alpha) T(0)<T(L) \rightarrow \infty$ as $\lambda \rightarrow \infty$.

From (13) it can be seen that for resistivity $1 / \sigma(T)$ like (8), with $T_{d}$ sufficiently small, $V$ is initially increasing as a function of $T(0)$, decreases, and then, eventually,

$$
V^{2} \sim 4 \kappa R_{\infty} T(0) L / \alpha
$$

for high temperatures; the response diagram is like that of Figure 2(left). The resistivity is a decreasing function of temperature (for paste) and, as in [3], it follows that the middle of the three branches, where $T$ is decreasing as a function of $V$, is unstable (regarding $V$ as fixed).

Without finding explicit or numerical solutions for given $R(T)$, the interval of $V$ corresponding to multiple solutions (with an unstable intermediate steady state) cannot be exactly identified - even whether or not it exists is not immediately clear. Since explicit solutions are hard to come by, numerical calculations would be needed and rather than do such exhaustive simulations, only a simple treatment, using something like (8),

$$
\sigma(T)=1 /\left(S_{1} e^{-T / T_{1}}+S_{2}\right)
$$

is carried out.

The problem (12), (15) can be made dimensionless by writing

$$
\begin{gathered}
z=L Z, T=T_{1} u, R=\frac{S_{2} F}{\mathcal{A}}, \mu=\lambda S_{2} L^{2} / T_{1} \mathcal{A}, a=\alpha / L, K=S_{1} / S_{2}: \\
\frac{d^{2} u}{d Z^{2}}+\mu r(u)=0, \quad a \frac{d u}{d Z}(0)=u(0), \quad \frac{d u}{d Z}(1)=0,
\end{gathered}
$$


with

$$
r(u)=K e^{-u}+1 .
$$

It should be first observed that if $a \ll 1$ (apparently a "long" electrode) the top boundary condition reduces to a Dirichlet one, $u(0)=0$. It has been found, [1], [3], that such problems with $V$ as the controlling parameter never have multiple solutions: $\int_{0}^{u(1)} r(u) d u$ increases monotonically with temperature $u(1)$.

At this point it is worth commenting on the effect of non-zero electrode velocity. Numerical simulations of this problem were carried out during the Study Group, taking a resistivity like (17), including a $\frac{d u}{d Z}$ term, representing convection, as well as the diffusive term $\frac{d^{2} u}{d Z^{2}}$, in the equation, and imposing the Dirichlet condition at $Z=0$. The voltage-temperature response diagram remained monotonic. (This is consistent with the purely convective problem, [4].) This suggested that the inclusion of convection was not significant as regards the qualitative behaviour so convection was not considered in the later models; convection will, of course, significantly affect quantitative results.

The contrasting limiting case, $\alpha \ll 1$ (which may be thought of as a short, fat electrode if $\alpha=$ electrode diameter) gives poor heat loss (the problem has approximate homogeneous Neumann conditions at both ends). This case has not been discussed but might, with appropriate limiting values of other parameters, lead to the lumped model of Section 4.

We now suppose that $a$ is neither large nor small: $\alpha$ is of order $L$.

From the earlier discussion of "small" $T_{d}$, it is clear that if $K$ is large enough (the smallest value of $\sigma$ is small enough compared with the hightemperature value), then there is a range of voltages for which there are three solutions. This voltage is, by (13), of size $\sqrt{K S_{2} T_{d}}$ (assuming that $S_{1}$ is not very much greater than $S_{2}$ ). The corresponding electric current is of magnitude $\frac{\mathcal{A}}{L} \sqrt{\frac{K T_{d}}{S}}$.

Following Section 4 , the electrode is now represented by a pair of onedimensional devices. The pair of temperature equations is

$$
\begin{aligned}
& \rho C \frac{\partial T_{1}}{d t}=\kappa \frac{\partial^{2} T}{\partial z^{2}}+\frac{J_{1}^{2} R\left(T_{1}\right)}{\mathcal{A}}-h\left(T_{1}-T_{2}\right), \\
& \rho C \frac{\partial T_{2}}{\partial t}=\kappa \frac{\partial T_{2}}{\partial z^{2}}+\frac{J_{2}^{2} R\left(T_{2}\right)}{\mathcal{A}}+h\left(T_{1}-T_{2}\right),
\end{aligned}
$$

where $\mathcal{A}$ is now the cross-sectional area of each half of the electrode and $h$ is some effective heat-transfer coefficient, expected to be of size $K / \mathcal{A}$, (such a representation really requires that the time scale $\gg \rho C \mathcal{A} / K$.) The currents 
through each half are determined by specifying total current $J$ :

$$
J_{1}+J_{2}=J
$$

and having the same potential drop:

$$
J_{1} \int_{0}^{L} R\left(T_{1}\right) d z=J_{2} \int_{0}^{2} R\left(T_{2}\right) d z
$$

It is clear that (18) - (21) have a symmetric steady state $\left(T_{1}=T_{2}, J_{1}=\right.$ $J_{2}=I \equiv J / 2$ ) as for the single-temperature model. The stability of such equilibria was then investigated.

Carrying out a linear stability analysis, writing

$$
J_{1}=I+j, \quad J_{2}=I-j
$$

to satisfy $(20)$,

$$
T_{1}=T+u, \quad T_{2}=t-u,
$$

where $T(z)$ is the solution of $(12)$, gives

$$
\rho C \frac{\partial u}{\partial t}=\kappa \frac{\partial^{2} u}{\partial z^{2}}+\frac{I^{2}}{\mathcal{A}} R^{\prime} u+2 \frac{I R}{\mathcal{A}} j-h u
$$

with

so

$$
I \int_{0}^{L} R^{\prime} u d z+j \int_{0}^{L} R d z=0
$$

$$
\rho C \frac{\partial u}{\partial t}=\kappa \frac{\partial^{2} u}{\partial z^{2}}+\frac{I^{2} R^{\prime} u}{\mathcal{A}}-\frac{2 I^{2} R \int_{0}^{L} R^{\prime} u d z}{\mathcal{A} \int_{0}^{L} R d z}-h u .
$$

This problem may be compared with that given by linearising the single equation with fixed $V$ :

$$
\rho C \frac{\partial u}{\partial t}=\kappa \frac{\partial^{2} u}{\partial z^{2}}+\frac{V^{2} R^{\prime} u}{\mathcal{A}\left(\int_{0}^{L} R d z\right)^{2}}-\frac{2 V^{2} R \int_{0}^{L} R^{\prime} u d z}{\left(\int_{0}^{L} R d z\right)^{3}} .
$$

On substituting current $I=V /\left(\int_{0}^{L} R d z\right)$ into (22), it is seen that (22) is just (23), with an extra, stabilising, term -hu.

It follows that for symmetric steady states corresponding to (10) having a single stationary solution, or indeed to any equilibrium which is increasing as a function of $V$, so that the results of [3] indicate stability for problem (10), this static situation is also stable for the more involved models (18) - (21). Taking $h$ to be small enough, the converse will hold, if the steady state corresponds to one which is a decreasing function of $V$ then (18) - 
(21) exhibits instability. Increasing $h$ has the effect of making smaller the range of $V$ and $J$ with unstable, symmetric, stationary solutions. In this unstable range it is possible to find stable steady states which are asymmetric: $T_{1}(z) \neq T_{2}(z)$. Fig. 5 shows the temperature profiles found for a particular example, with a $\sigma$ as in (15), and including a convective term.

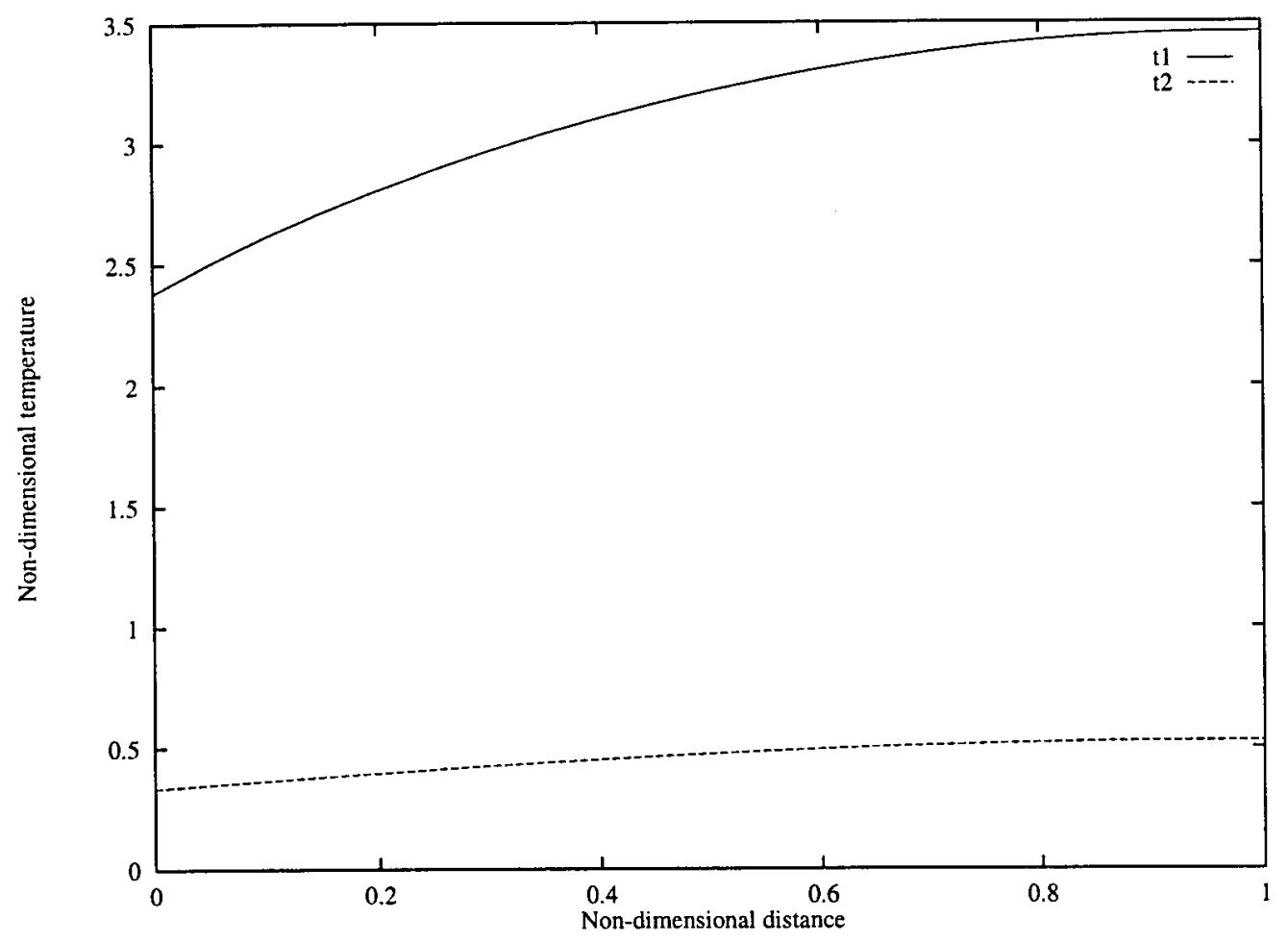

Figure 5: Asymmetric temperatures with $\rho C U L / \kappa=1, R(T)=R_{\infty} e^{-T / T_{d}}$, $\alpha / L=1, h L^{2} / \kappa=0.1$, and $J^{2} L^{2} R_{\infty} / \kappa T_{d} \mathcal{A}=100$.

The linear instability shown by (23) is associated with the positivity of the largest eigenvalue $\gamma$ for

$$
\rho C \gamma \psi=\kappa \frac{d^{2} \psi}{d z^{2}}+\frac{I^{2} R^{\prime} \psi}{\mathcal{A}}-\frac{2 I^{2} R \int_{0}^{L} R^{\prime} \psi d z}{\mathcal{A} \int_{0}^{L} R d z}
$$

given by writing $u=e^{\gamma t} \psi(z)$. The coupled problem is unstable if $h<\gamma$. Looking at the size of the last term of (24) (again using (15)), as this is the only destabilising term, indicates that instability might only happen if $\kappa$ is small enough compared with $S_{2} J^{2} / \mathcal{A} T_{d}$.

As a final generalisation of this one-dimensional model a two-dimensional version, with a one-dimensional steady state, can be considered. (A threedimensional case, say a circular cylinder, could be discussed with little additional difficulty. The qualitative results are expected to be the same.) 
The pair (18), (19) is replaced by

$$
\begin{gathered}
\rho C \frac{\partial T}{\partial t}=\kappa\left(\frac{\partial^{2} T}{\partial y^{2}}+\frac{\partial^{2} T}{\partial z^{2}}\right)+\sigma(T)|\nabla \varphi|^{2} \\
\nabla \cdot(\sigma(T) \nabla \varphi)=0
\end{gathered}
$$

(which is the two-dimensional version of (4) - (5) with no convection).

The boundary conditions are

$$
\varphi=0 \text { on } z=0, \quad \varphi=V(t) \text { on } z=L \text { such that }\left.\ell \int_{0}^{\ell}\left(\sigma \frac{\partial \varphi}{\partial z}\right)\right|_{z=L} d y=J
$$

(the cross-section is $0<x<\ell, 0<y<\ell$ and $x$ variation is not considered),

$$
\begin{gathered}
\frac{\partial \varphi}{\partial y}=0 \text { on } y=0 \text { and on } y=\ell, \\
\alpha \frac{\partial T}{\partial z}=T \text { on } z=0, \frac{\partial T}{\partial z}=0 \text { on } z=l, \\
\frac{\partial T}{\partial z}=0 \text { on } y=0 \text { and on } y=\ell .
\end{gathered}
$$

The basic steady states, $T=T(z), \varphi(z)=I \int_{0}^{z} d z / \sigma(T(z))$ are the same as for the simple one-dimensional model $\left(I=J / \ell^{2}\right)$. Now a suitable linear perturbation, to satisfy the conditions on $y=0$ and $y=\ell$, is

$$
\begin{gathered}
u(z, t) \cos \pi y / \ell \text { for temperature } \\
v(z, t) \cos \pi y / \ell \text { for potential. }
\end{gathered}
$$

The top and bottom boundary conditions for temperature hold if $\alpha \frac{\partial u}{\partial z}=u$ on $z=0$ and $\frac{\partial u}{\partial z}=0$ on $z=L$. The remaining boundary conditions, for potential, require $v=0$ on $z=0$ and $z=L$. The total current condition is automatically satisfied.

The linearised equations are:

$$
\begin{gathered}
\rho C \frac{\partial u}{\partial t}=\kappa \frac{\partial^{2} u}{\partial z^{2}}-\frac{\pi^{2} \kappa}{\ell^{2}} u+\left(\frac{d \varphi}{d z}\right)^{2} \sigma^{\prime} u+2 \frac{d \varphi}{d z} \sigma \frac{\partial v}{\partial z} \\
\text { or } \rho C \frac{\partial u}{\partial t}=\kappa \frac{\partial^{2} u}{\partial z^{2}}-\frac{\pi^{2} \kappa}{\ell^{2}} u+I^{2} \sigma^{-2} \sigma^{\prime} u+2 I \frac{\partial v}{\partial z} \\
\text { and } \frac{\partial}{\partial z}\left(\sigma \frac{\partial v}{\partial z}+\sigma^{\prime} \frac{d \varphi}{d z} u\right)-\frac{\pi^{2}}{\ell^{2}} v=0 .
\end{gathered}
$$




$$
\text { or } \frac{\partial}{\partial z}\left(\sigma \frac{\partial v}{\partial z}+I \sigma^{-1} \sigma^{\prime} u\right)-\frac{\pi^{2}}{\ell^{2}} v=0 .
$$

The problem of finding whether the solution $(u, v)$ of $(27),(28)$ is growing or decaying (for $u=e^{\gamma t} \psi\left(z_{l}\right), v=e^{\gamma t} \chi(z), \gamma>0$ or $\gamma<0$ respectively) is still far from trivial. Two limiting cases can be taken further. Firstly, for $\ell \ll L$ (a long or thin electrode), the second term on the right-hand side of (27) is clearly important and indicates stability. Secondly, for $\ell \gg L$ (a short or fat electrode), the second term on the right-hand side of (27) and the last term in (28) can be neglected to give

$$
\begin{gathered}
\rho C \frac{\partial u}{\partial t}=\kappa \frac{\partial^{2} u}{\partial z^{2}}+I^{2} \sigma^{-2} \sigma^{\prime} u+2 I \frac{\partial v}{\partial z} \\
\text { and } \frac{\partial}{\partial z}\left(\sigma \frac{\partial v}{\partial z}+I \sigma^{-1} \sigma^{\prime} u\right)=0
\end{gathered}
$$

Integrating (30) gives

$$
\sigma \frac{\partial v}{\partial z}+I^{-1} \sigma \sigma^{\prime} u=j(y, t)
$$

so $\frac{\partial v}{\partial z}=j / \sigma-I \sigma^{-2} \sigma^{\prime} u$, which, since $v=0$ at both ends of the electrode, means that $j=I \int_{0}^{L} \sigma^{-2} \sigma^{\prime} u d z / \int_{0}^{L} \sigma^{-1} d z$ and

$$
\frac{\partial v}{\partial z}=I\left(\frac{\int_{0}^{L} \sigma^{-2} \sigma^{\prime} u d z}{\sigma \int_{0}^{L} \sigma^{-1} d z}-\sigma^{-2} \sigma^{\prime} u\right) .
$$

Substitution of this into (29) yields

$$
\rho C \frac{\partial u}{\partial t}=\kappa \frac{\partial^{2} u}{\partial z^{2}}-I^{2} \sigma^{-2} \sigma^{\prime} u+\frac{2 I^{2} \int_{0}^{L} \sigma^{-2} \sigma^{\prime} u d z}{\sigma \int_{0}^{L} \sigma^{-1} d z},
$$

which, on taking the appropriate relations between $V$ and $I$ (also $R$ and $\sigma^{-1}, R^{\prime}$ and $\sigma^{-2} \sigma^{\prime}$ ) is just (23). This indicates, once again, that if the basic one-dimensional solution corresponds to an intermediate (unstable) solution for fixed $V$, then it can be unstable, even for fixed current $J$, if the aspect ratio is large enough.

\section{Another One-Dimensional Model}

A related and reasonably simple situation is to have an electrode with dominant heat flow across it: the ends are effectively insulated so $\frac{\partial T}{\partial z} \simeq 0$ at each. 
Once again it is possible to obtain a single, but nonlocal, equation modelling this case (again see [1]). By relating electric field $V / L$ to total current $J$ and local current density $j\left(j=\sigma V / L\right.$ in the $z$ direction, $J=(V / L) \int_{\Omega} \sigma d x d y$ if $\Omega$ is the cross-section of the electrode), temperature is seen to satisfy

$$
\rho C \frac{\partial T}{\partial t}=\kappa\left(\frac{\partial^{2} T}{\partial x^{2}}+\frac{\partial^{2} T}{\partial y^{2}}\right)+\frac{J^{2} \sigma}{\left(\int_{\Omega} \sigma d x d y\right)^{2}} .
$$

Considering the one-dimensional version of this,

$$
\rho C \frac{\partial T}{\partial t}=\kappa \frac{\partial^{2} T}{\partial y^{2}}+\frac{J^{2} \sigma}{\left(\int_{0}^{\ell} \sigma d x d y\right)^{2}},
$$

the steady states take the form $T=T(y)$. For special cases $\sigma(T)=S_{3} e^{T / T_{d}}$ or $\sigma=S_{3}+S_{4} T$ results of [5] show that these stationary solutions are stable to perturbations independent of $x$ and $z$. It is expected that similar results hold for more general conductivities $\sigma(T)$ and for $z$-independent perturbations of equilibria of (31). This would mean that this simple configuration would allow no instabilities independent of height in the electrode; more general perturbations $(e . g ., u(x, y, z, t))$ have yet to be considered.

\section{Alternating Current}

On taking the electric field to be $\operatorname{Re}\left\{\boldsymbol{E} e^{i w t}\right\}$ (the complex exponential accounts for the alternating field; $\boldsymbol{E}$ might also vary over a slower time scale, the thermal time scale) and neglecting electrical permittivity, Maxwell's equations give

$$
\nabla^{2} \boldsymbol{E}-\nabla(\nabla \cdot \boldsymbol{E})=i \mu \omega \sigma \boldsymbol{E} \text { with } \nabla \cdot(\sigma \boldsymbol{E})=0 .
$$

These equations are coupled with the heat equation

$$
\rho C\left(\frac{\partial T}{\partial t}+U \frac{\partial T}{\partial z}\right)=\kappa \nabla^{2} T+\sigma|\boldsymbol{E}|^{2} .
$$

This more complete problem still requires attention.

There was a suggestion that the oscillations would result in a skin effect with nearly all the current flowing near the surface of the electrode. Calculations done in the course of the 1997 Study Group indicated that this was unlikely for carbon paste. If this effect were present then the analysis of Section 5, and possibly of Section 6, could still have relevance, taking the effective conductivity to be $\sigma \times$ skin thickness (which should be $\propto 1 / \sqrt{\sigma}$ ). Care would need to be taken with regard to how the heat-transfer terms also vary with skin width. 


\section{References}

[1] A.A. Lacey, Thermal runaway in a non-local problem modelling Ohmic heating. I: Model derivation and some special cases, Eu. Jl. Appl. Maths. 6, 1995, 127-144.

[2] N. Chafee, The electrical ballast resistor: homogeneous and nonhomogenous equilibria. In Nonlinear Differential Equations: Invariance, Stability and Bifurcations, eds. P de Mottoni and L Salvadori, 97 - 127, Academic Press, New York, 1981.

[3] A.A. Lacey, Thermal runaway in a non-local problem modelling Ohmic heating. II: General proof of blow-up and asymptotics of runaway, Eu. Jl. Appl. Maths. 6, 1995, 201-224.

[4] A.A. Lacey, D.E. Tzanetis and P.M. Vlamos, Behaviour of a non-local reactive convective problem modelling Ohmic heating of foods, preprint.

[5] P. Freitas, A non-local Sturm-Liouville eigenvalue problem, Proc. Roy. Soc. Ed., 124A, 1994, 169 -188.

Report submitted by Andrew Lacey.

Contributions from: Harald Hanche-Olsen, Peter Howell, Andrew Lacey, Mark Peletier, Donald Schwendeman, Warren Smith, Richard Tew, Jonathan Wattis. 
